\title{
Mortalidad Perinatal Estudio Cooperativo Institucional (Informe Preliminar)
}

\author{
Dres.: Germán Uriza Gutiérrez y Jairo Barragán Espinosa
}

PONENCIA OBSTETRICA DEL

$X V I$ CONGRESO COLOIMBIANO DE OBSTETRICIA Y GINECOLOGIA

\section{INVESTIGADORES INSTITUCIONALES}

HOSPITALES UNIVERSITARIOS:

$\begin{array}{ll}\text { Hospital Materno Infantil } & \text { Doctor Arturo Rodríguez } \\ \text { Hospital Militar Central } & \text { Doctor Jairo de la Cruz } \\ \text { Hospital de la Samaritana } & \text { Doctor Luis E. Pontón } \\ \text { Hospital San Ignacio } & \text { Doctor Jairo Barragán } \\ \text { Hospital San José } & \text { Doctor Germán Montoya } \\ \text { Hospital Infantil } & \text { Doctor Jesús Gómez }\end{array}$

CLINICAS PRIVADAS:

Clínica David Restrepo Doctor Carlos Alviar

Clínica Palermo Doctor Saúl Santoyo

Centro Médico de los Andes Doctor Jorge Medina

SECRETARIA DE SALUD DISTRITAL:

Hospital Simón Bolívar

Doctor Alfonso López

Hospital San Blas

Doctor Guillermo González

CENTRO DE COMPUTO DE LA

UNIVERSIDAD NACIONAL DE COLOMBIA

Doctor Ramón Díaz

Ingeniero Carlos Briceño

PROGRAMA PAMI UNIVERSIDAD JAVERIANA

Estadístico

Señor Germán León Pieschacón 


\section{1 - INTRODUCCION Y JUSTIFICACION}

Existe el convencimiento por parte de importantes investigadores, de que la mortalidad perinatal constituye uno de los indicadores más sensibles de la calidad del proceso reproductivo (27).

La Federación Colombiana de Sociedades de Obstetricia y Ginecología, conciente de ésta verdad y ante la poca Bibliografía Colombiana que sobre este tema se tiene y como consecuencia el desconocimiento de la situación real en nuestro país, decidió durante el $X V$ Congreso Colombiano de la especialidad que se realizó en Bucaramanga, tomar como uno de los temas oficiales para el XVI Congreso a celebrarse en Cali, la Mortalidad Perinatal.

La menor tasa de Mortalidad Perinatal encontrada en los países industrializa dos, que en algunos ha alcanzado ya la cifra de $10 \times 1000$ (27), comparada con la mayor tasa de los países subdesarrollados (Tabla 1) ha sido demostrada plenamente por Barns (4).

Los factores de tipo social como: menor estatus socioeconómico, baja escolaridad y falta de compañero permanente son factores que inciden en el hallazgo de una mayor tasa de mortalidad perinatal en cualquier pa is donde se realice este tipo de investigaciones (1) (13) (14) (15) (19) (20) (23) (25) (27) (30) (34).

La OMS consignó en 1970, siete veces mayor mortalidad fetal tardía y cuatro veces mayor mortalidad neonatal temprana (mortalidad perinatal de nuestro estudio), en los países subdesarrollados o en vías de desarrollo, que en los países desarrollados o industrializados (3).

TABLA 1

TASAS DE MORTALIDAD COMPARATIVA SEGUN DIFERENTES AUTORES

\begin{tabular}{|c|c|c|c|c|}
\hline REF & AUTOR & ORIGEN & Ño & TASA $\times 1.000$ \\
\hline 1 & ABUDU 0. & LAGOS UNIVERSITY & '72-'82 & 42.5 \\
\hline 9 & $D I A Z R$. & SECRETARIA SALUD DISTRITAL & '83-84 & 29.7 \\
\hline 29 & LOPEZ G. & PARTO HOSPITAL ARIO EN COLOMBIA & 79 & 28.9 \\
\hline 45 & TERRIN M. & ONTARIO CANADA (FUMADORAS) & $60-61$ & 28.2 \\
\hline 23 & LENGER A. & IMPER MEXICO & '83 & 26.2 \\
\hline 45 & TERRIN M. & ONTARIO CANADA (NO FUMADORAS) & '60-'61 & 22.0 \\
\hline 21 & KNUT ZEN V. & NUEVA YORK & '75-78 & 20.0 \\
\hline 36 & PEARSUN J. & INDIANA UNIVER & '82 & 18.0 \\
\hline 33 & O' DRIS COLL & DUBLIN & ' 80 & 16.8 \\
\hline 5 & BOYLAN P. & DUBLIN & '83 & 16.0 \\
\hline 11 & ERKKOLA & FINLANDIA & $70-75$ & 15.7 \\
\hline 25 & LEE K. & AMERICAN COLLEGE (OBJETIVO) & '76 & 10.0 \\
\hline 11 & ERKKOLA & FINLANDIA & $76^{2} 78$ & 8.9 \\
\hline
\end{tabular}

MORTALIDAD PERINATAL, ESTUDIO COOPERATIVO S. C. 0. 6. -1985 (REVISION BIBLIOGRAFICA 
Hay estudios que afirman que para los países del tercer mundo en vías de desarrollo o subdesarrollados, es mucho más importante un adecuado y cuidadoso control prenatal para reducir la tasa de mortalidad perinatal, que lo que se pueda lograr con las costosas y sofisticadas Unidades de Cuidado Intensivo Neonatal, que vienen a agravar la ya precaria situación económica de sus instituciones hospitalarias (13). Las Unidades de Cuidado Intensivo Neonatal sí juegan un papel fundamental en los países industrializados, como lo demuestran otros autores (41) (43). Pensamos que las dos posiciones son igualmente importantes $y$ que lo más adecuado como lo afirma otro autor, es lograr la regionalización de la atención perinatal con el propósito de atender los pacientes de bajo riesgo en el Nivel 1 o inferior $y$ las de alto riesgo en el Nivel III o superior, dotando a este nivel de alta complejidad, de todos los elementos que les permitan establecer unidades de Cuidado Intensivo Neonatal (4). La regionalización adquiere su primera importancia, en la medida en que esta se cumpla estrictamente, pues mientras los centros del Nivel II sigan en nuestro país atendiendo en su mayoría pacientes de bajo riesgo, la regionalización pierde todo su fundamento.

El factor más directamente relacionado con la mayor tasa de mortalidad perinatal es el menor peso al nacer, $y$ de todos es conocido, que los recién nacidos de los países del tercer mundo tienen un peso menor que el de los nacidos en los países industrializados, y cómo la mortalidad perinatal es cada vez mayor, cuando menos sea el peso al nacimiento (1) (11) (21) (23) (36) (44). En relación con el peso al nacimiento hay un estudio curioso, que muestra como la mortalidad perinatal de un recién nacido en un parto sucesivo es mayor, si el primer nacido fue más pesado que el segundo; y la tendencia es a aumentar cuando la diferencia de peso entre los dos es mayor (26).
듸 trabajo de parto pretérmino es un factor etiológico fundamental, que contribuye con gran frecuencia a una mayor tasa de mortalidad perinatal, ya que se encuentra íntimamente relacionado con el bajo peso al nacer (5) (17) (21) (37). El problema obstétrico más común asociado con el trabajo de parto pretérmino, es la ruptura prematura de membranas, que además expone al producto y a la madre al riesgo de infección, dependiendo la sobrevida de ambos en estos casos de un adecuado manejo obstétrico del problema (8) (16) (37). Otros factores importantes de iniciación de trabajo de parto pretermino $y$ de recién nacidos de bajo peso, es el embarazo general (19) (38) (45); un estudio sobre mortalidad perinatal muestra una tasa de $112.3 \times$ 1000 para los gemelares, comparada con $15 \times 1000$ para los simples (41).

Se ha sugerido que el aumento de la cesárea en la práctica de la Obstetricia moderna, que en los Estados Unidos ha pasado del $5 \%$ en 1965 , al $15 \%$ en 1980 , es el factor que más ha influido en la disminución de la mortalidad perinatal; sin embarqo, la revisión de 108.987 nacimientos en la Maternidad Nacional de Dublín, muestra un porcentaje de cesárea prácticamente igual en los últimos 15 años; en 1965 de 4.2, 1970 de 4.2, 1975 de 4.2 y en 1980 de 4.8 ; con una continua reducción de la tasa de mortalidad perinatal para los mismos años de: $42.1 \times 1000$ en $1965,36.5 \times 1000$ en 1970,24 × 1000 en 1975 y $16.8 \times 1000$ en 1980 (33). Este estudio demuestra que hay otros factores relacionados con la atención perinatal, más importantes que la sola cesárea en la reducción de la mortalidad y que la monitoría ante parto e intraparto externa, sin el apoyo de la medición del $\mathrm{PH}$ en el cuero cabelludo fetal, pueda ser responsable del aumento en el número de cesárea en los Estados Unidos y otros países (36).

Otros autores señalan que el parto por cesárea en fetos de menos de 2.000 
gramos, disminuye en forma sensible la mortalidad perinatal en este grupo de recién nacidos (46), siendo especialmente importante en los casos de presentación de pelvis; sin embargo, otros autores demuestran igual reducción en recién nacidos de bajo peso, tanto en presentación de pelvis como en presentación de vértice, sugiriendo que el aumento en el número de ceśareas es un hallazgo más bien incidental, que causal (10).

La monitoría selectiva intraparto ha demostrado ser de gran beneficio con el fin de disminuir la morbilidad y la tasa de mortalidad perinatal, especialmente en recién nacidos de bajo peso, $(1.500$ gramos o menos) (2) (35), y en todos los casos de embarazos de post-término; siendo la causa más frecuente de muerte en este grupo de pacientes, la hipoxia perinatal por masiva aspiración de líquido amniótico y de meconio, y en segundo lugar las malformaciones congénitas incompatibles con la vida (44).

En los casos de fetos a término e hipertróficos, el factor asociado más frecuentemente con la mortalidad perinatal. que es baja en este grupo, es el trauma obstétrico, el cual podría evitarse, estableciendo el diagnóstico de desproporción cefalo pélvico a tiempo, con el fin de practicar la operación cesárea que evite el trauma obstétrico (1) (23) (33).

Existen otros factores menos frecuentes pero plenamente conocidos como causales de una mayor tasa de mortalidad perinatal; unos de origen fetal como: Anomalías congénitas (1) (6) (14) (28) (38) (48), embarazos múltiples (19) (38) (41), macrosomía (24) (48), retardo del crecimiento intra-uterino (21) (47) y eritroblastosis (21). Otros de origen materno como: Enfermedades hereditarias (19), abruptio placentae (31), placenta previa (31), hemorragia (1), pre eclampsia (1) (34) y diabetes (24) (48).
Otros factores maternos más frecuentes, también asociados a una mayor tasa de mortalidad perinatal son: la gran multiparidad (42), la bacteriuria asintomática y la infección urinaria (5) (20), el embarazo en adolescentes (14) (15) (17) (19) (30) (42), o en mayores de 35 (15) (19) (42).

Algunos factores asociados a mayor tasa de mortalidad perinatal, que son menos frecuentes y también menos conocidos han sido reportados por otros autores: de origen infeccioso sistémico, como el Estreptococo Beta (6); o afectando el tracto genital como el Mycoplasma Hominis (6) (20), que en algunos casos puede colonizar la placenta (22); la Clamidia Tracomatis (6) (30) y la Gardnerella Vaginalis (6). De origen externo como: El cigarrillo (12) (31) (34) (45), o la ingestión de drogas de tipo de la Indometacina utilizada en el tratamiento de la amenaza de parto pretermino y asociada con Oligoamnios, meconio v muerte perinatal (18).

Finalmente es necesario en los casos de pacientes de alto riesgo, la creación de clinicas que manejen exclusivamente a estos pacientes (24), siendo fundamental para disminuir la muerte neonatal precoz y tardía del recién nacido del alto riesgo, el papel de la familia, especialmente el soporte de la madre y el padre.

\section{2 - MATERIAL Y METODOS}

\subsection{Propósito y Ohjetivos:}

2.1.1 Objetivo General: Desarrollar una metodología para el estudio de la Mortalidad Perinatal Institucional en Bogotá, con el propósito de que la misma pueda extenderse a todo el país, y de esta manera lograr un conocimiento más exacto de uno de los indicadores más importantes de la Salud Perinatal. 


\subsubsection{Objetivos Específicos:}

2.1.2.1 Identificar la magnitud del problema.

2.1.2.2 Identificar las causas de mortalidad perinatal.

2.1.2.3 Presentar la metodología, los parámetros del estudio y los resultados de la investigación en el XVI Congreso Colombiano de la Especialidad.

\subsection{Tipo de Investigación}

Estudio prospectivo y cooperativo sobre mortalidad Institucional en 11 Centros de Bogotá.

\subsection{Recolección de la Información}

Se tomaron todas las muertes perinatales ocurridas en las 11 instituciones de Bogotá, desde febrero $1 / 85$ a julio 31 / 85 , utilizando para la recolección de la información, el formulario de Registro de Defunción Perinatal modificado del IFRP (International Fertility Research Program) hoy IHF (International Health Family) de North Carolina, previa autorización de la CCRP (Corporación Centro Regional de Población) de Bogotá, (Anexo 1).

Se diseñó un instructivo para recolectar correctamente la información (Anexo 2).

El total de muertes perir stales fue de 405 sobre los cuales se caiculó la tasa. Los formularios se corrigieron, se verificaron las inconsistencias y se eliminaron algunos, quedando para el análisis 392 casos.

\subsection{Procesamiento de la Información}

Los casos del estudio fueron procesados en el Centro de Cómputo de la Universidad Nacional, bajo la dirección del Doctor Ramón Díaz y con la colaboración del Ingeniero Carlos Briceño.

\subsection{Instituciones Participantes}

Participaron en el estudio 11 instituciones de Bogotá, con la coordinación general del Doctor Germán Uriza, que se enumeran a continuación:

$\begin{array}{cll}\text { Código } & \text { Institución } & \text { Coordinador } \\ 01 & \text { Hospital Materno Infantil } & \text { Dr. Arturo Rodríguez } \\ 02 & \text { Hospital Militar Central } & \text { Dr. Jairo de la Cruz } \\ 03 & \text { Hospital Simón Bolivar } & \text { Dr. Alfonso López } \\ 04 & \text { Hospital San Ignacio } & \text { Dr. Jairo Barragán } \\ 05 & \text { Hospital San Blas } & \text { Dr. Guillermo González } \\ 06 & \text { Clínica Palermo } & \text { Dr. Saúl Santoyo } \\ 07 & \text { Hospital San José } & \text { Dr. Germán Montoya } \\ 08 & \text { Hospital Infantil } & \text { Dr. Jesús Gómez } \\ 09 & \text { Centro Médico de los Andes } & \text { Dr. Jorge Medina } \\ 10 & \text { Clínica David Restrepo } & \text { Dr. Carlos Alviar } \\ 11 & \text { Hospital La Samaritana } & \text { Dr. Luis E. Pontón }\end{array}$

\subsection{Glosario de Términos}

Se utilizaron las definiciones y fórmulas empleadas por el CLAP (Centro Latinoamericano de Perinatología y Desarrollo Humano) y que a continuación se describen (39) (Cuadro 1).

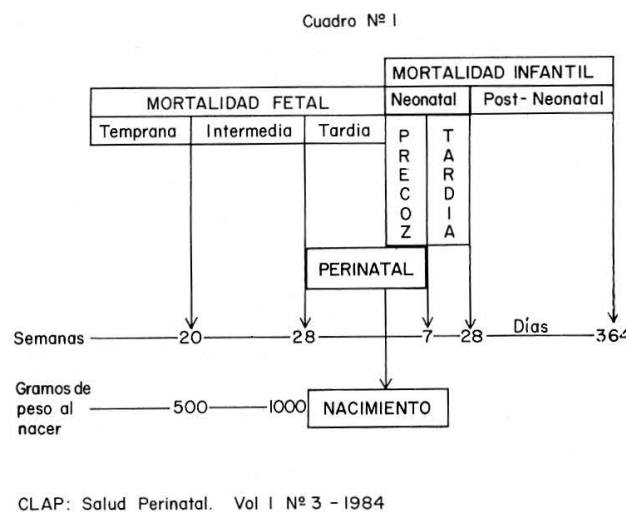

2.6.1 Mortalidad Perinatal: Toda muerte del producto ocurrida a partir de la semana 28 de gestación y hasta el 7o. día de nacido. En caso de no conocimiento de la edad gestacional se utilizó como parámetro todo producto muerto de 1.000 gramos de peso o más. 
2.6.2 Mortalidad Fetal: Toda muerte de producto desde el inicio del embarazo hasta el nacimiento.

2.6.3 Mortalidad Fetal Temprana (Aborto): Toda muerte del producto desde el inicio del embarazo hasta la semana 20.

2.6.4 Mortalidad Fetal Intermedia: Toda muerte del producto desde la semana 21 de gestación hasta la semana 27.

2.6.5 Mortalidad Fetal Tardia: Toda muerte del producto desde la semana 28 de gestación hasta inmediatamente antes del nacimiento (incluyendo muerte intraparto).

2.6.6 Mortalidad Infantil: Toda muerte del producto desde el nacimiento hasta los 364 días de nacido.

2.6.7 Mortalidad Neonatal: Toda muerte del producto desde el nacimiento hasta los 28 días de nacido.

2.6.8 Mortalidad Neonatal Precoz: Toda muerte del producto desde el nacimiento hasta el 7o. día de nacido.

2.6.9 Mortalidad Neonatal Tardia: Toda muerte del producto desde el 80. día de nacido hasta el día 28 de nacido.

2.6.10 Mortalidad Post-Neonatal: Toda muerte del producto desde el día 29 de nacido hasta el día 364 de nacido. (40).

\subsubsection{Tasa de Mortalidad Perinatal:}

Número de Mortinatos de 1.000 o más gramos más número de muertos de 1.000 o más gramos antes de los 8 días de nacidos.

$\mathrm{TMP}=$ $\times 1.000$

Número total de nacimientos vivos y muertos de 1.000 o más gramos al nacer.

\subsection{Análisis de la Información:}

Para este efecto se van a medir, la magnitud del problema por medio de la tasa de mortalidad perinatal y el establecimiento del valor porcentual de las causas de mortalidad perinatal.

\section{3- RESULTADOS}

\subsection{Tasa de Miortalidad Perinatal:}

La mayor tasa encontrada fue en el Instituto Materno Infantil con $34.25 \times$ 1.000 y la menor en el Hospital San Blas con $4.5 \times 1.000$. La tasa general para todas las instituciones fuje de $18.09 \times$ 1.000. (Tabla No. 2).

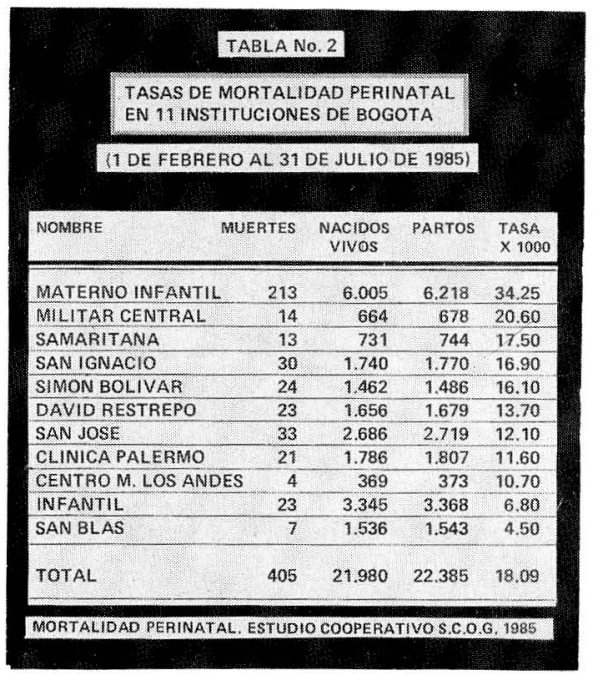

La tasa agrupada por tipos de institución fue para los Hospitales Universitarios de $21.03 \times 1.000$ (Tabla No. 2A); para las clínicas privadas de $12.43 \times$ 1.000 (Tabla No. 2B) y para las instituciones del Distrito Especial de Bogotá de $10.23 \times 1.000$ (Tabla No. 2 C).

\subsection{Edad Materna:}

Se trata de una población joven, con el mayor porcentaje en el grupo de 21 a 25 
años (31.90\%) y en segundo lugar el grupo de 16 a 20 años (25.25\%) (Gráfico 1 ).

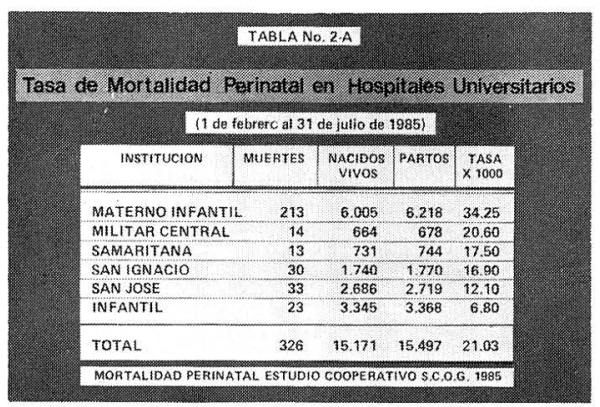

\begin{tabular}{|c|c|c|c|c|}
\hline \multicolumn{5}{|c|}{ TABLA NO. 2 . } \\
\hline \multicolumn{5}{|c|}{ Tasa de Mort. Perinatal en Clincas Privadas } \\
\hline \multicolumn{5}{|c|}{ (11 de fehrero al 31 de julio de 1085). } \\
\hline INSTITUCION & MUERTES & $\begin{array}{c}\text { Nacidos } \\
\text { Vivos }\end{array}$ & PARTOS & $\begin{array}{r}\text { TASA } \\
\times 1000 \\
\end{array}$ \\
\hline DAVID RESTREPO & 23 & 1.566 & 1.670 & 1370 \\
\hline PALEFMO & 21 & 1.786 & 1.807 & 11.60 \\
\hline CENTRO M. LOS ANDES & 4 & 369 & 373 & 10.70 \\
\hline TOTAL & 48 & 3.811 & 3.859 & 12.43 \\
\hline
\end{tabular}

\begin{tabular}{|c|c|c|c|c|}
\hline \multicolumn{5}{|c|}{ Tasa de Mort Perinatal en Hoso del Distrito } \\
\hline \multicolumn{5}{|c|}{ (1 de febrero a 31 de julio de 1985$)$} \\
\hline INSTITUCION & MUERTES & $\begin{array}{c}\text { NACIDOS } \\
\text { VIVOS }\end{array}$ & PARTOS & $\begin{array}{l}\text { TASA } \\
\times 1000\end{array}$ \\
\hline SIMON BOLIVAR & 24 & 1.462 & 1.486 & 16.10 \\
\hline SAN BLAS & 7 & 1,536 & 1.543 & 4.50 \\
\hline TOTAL & 31 & 2.998 & 3.029 & 10.23 \\
\hline
\end{tabular}

Agrupando las edades en adolescentes (menores de 18 años), de 18 a 34 y mayores de 35 , se encuentra un porcentaje importante en los dos extremos de la vida reproductiva: $8.41 \%$ en adolescentes y $13.5 \%$ en mayores de 35 años. La mayoría se encuentra en el grupo de 18 a 34 años (78.07\%) (Gráfico $1 \mathrm{~A}$ ).
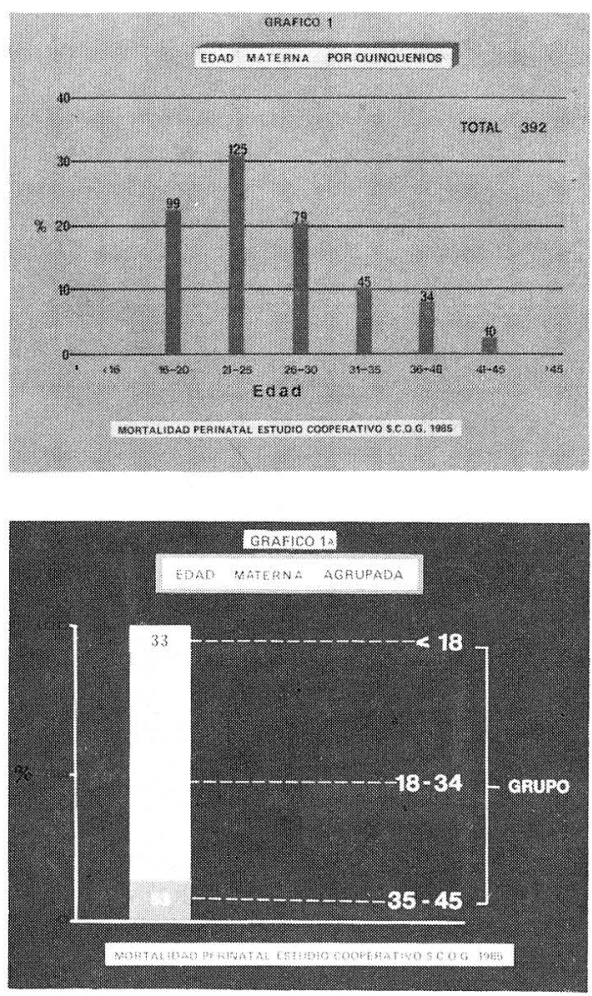

\subsection{Paridad:}

La mayoría de las pacientes son primigestantes con 165 casos (42.09\%); y las pacientes con antecedente de un parto 90 casos (22.96\%) (Gráfico 2).

Agrupando las pacientes en nulíparas, multíparas y grandes multíparas, se aprecia como la mayoría fueron multíparas, 208 casos $(53.07 \%$ con muy pocas grandes multíparas, 19 casos (4.84\%) (Gráfico $2 \mathrm{~A}$ ).

\subsection{Peso al Nacer:}

Los 4 casos de menos de 1.000 gramos cumplían los parámetros del estudio por edad gestacional. El grupo más numeroso de casos se encontró en los pre- 
maturos de 1.000 a 2.000 gramos, con 155 casos (39.54\%) y los de 2.001 a 2.499 gramos, con 60 casos (15.31\%) (Gráfico 3). Agrupando los casos en bajo peso y peso adecuado, se encuentra la mayoría, 219 casos con peso menor de 2.500 gramos (55.87\%) y 144 casos con peso adecuado (36.73\%) (Gráfico 3A).
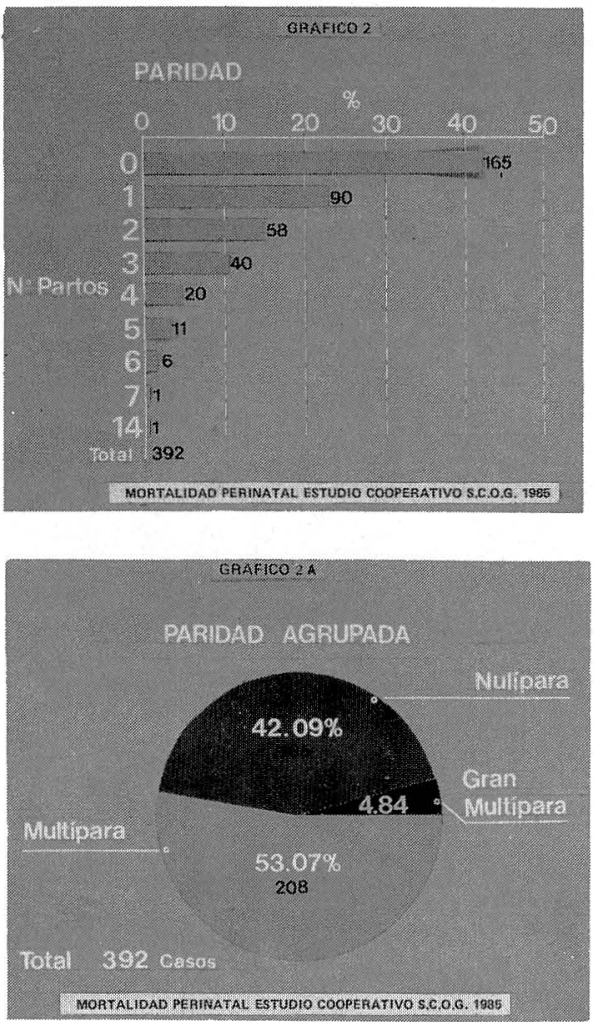

\subsection{Edad Gestacional:}

Fue desconocida en 17 casos; de los 375 casos de edad conocida, un gran número de ellos fueron pretermino 149 (39.73\%), con un número importante de post-término 50 (13.34응).

La mayoría de casos fueron a término 176 (46.93\%). Sin embargo, al sumar los recién nacidos con edad gestacional pretérmino más los post-término hacen mayoría, 199 (53.07은 (Gráfico 4).
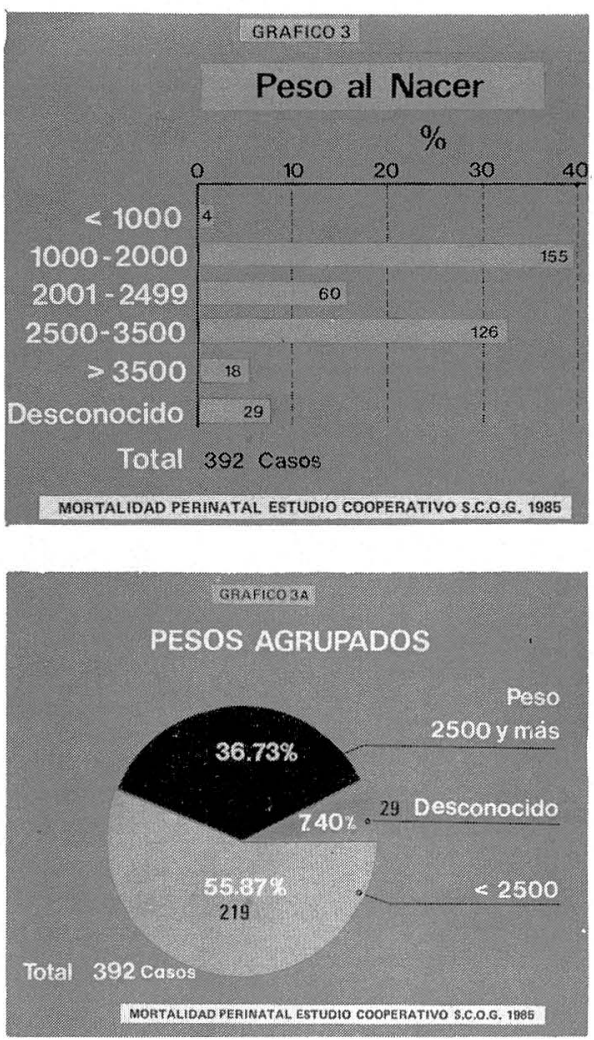

\subsection{Causa de Muerte Fetal por Autopsia}

Un gran número de casos quedaron sin autopsia, 191 (48.72\%). De los 201 casos a los cuales se les practicó autopsia (51.28\%), sólo se conoció el resultado de la misma en 39 casos (9.95\%) (Gráfico 5).

Los resultados de las autopsias se pueden ver en la Tabla 3, siendo las causas más frecuentes las anomalías congénitas con 9 casos (23.07\%), la anoxia 
intrauterina 9 casos (23.07\%) y 7 casos de maceración sin mayor información (17.94\%).
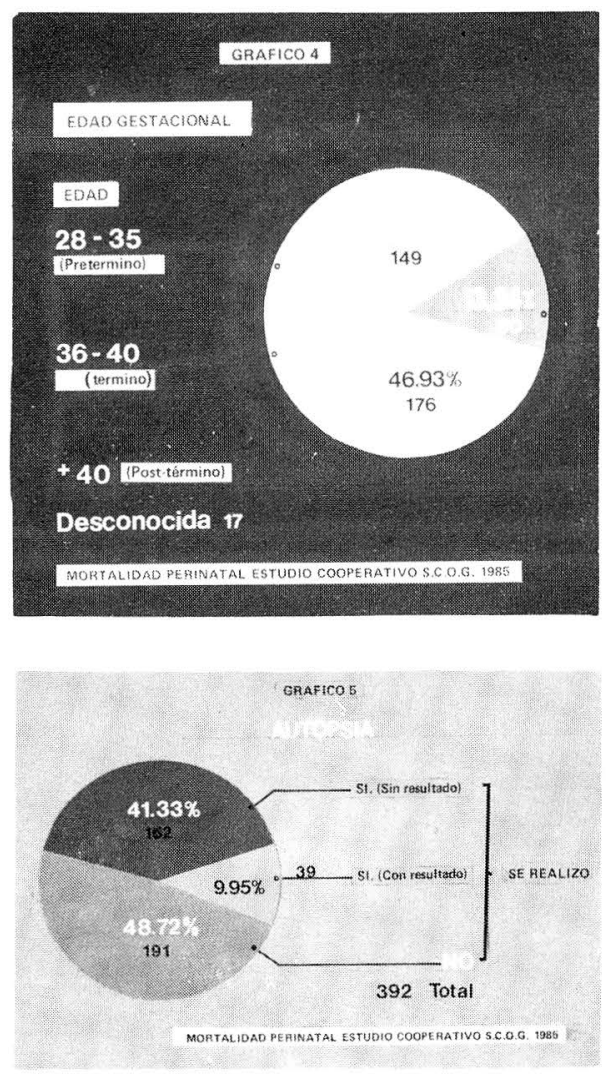

\subsection{Principal causa de Muerte Perinatal}

La principal causa conocida fue la as fixia del recién nacido, 76 casos (19.40\%); siquiendo en frecuencia la alteración placentaria, 39 casos (9.95\%); la altera ción del cordón umbilical, 35 casos (8.92) y la infección, 32 casos (8.16? (Tabla 4).

Se encuentran 232 casos de muerte intra-uterina (59.18\%), en su mayoría de causas desconocidas con 120 casos (51.72\%). De los casos en que se puede aclarar la causa, las principales fueron: la alteración placentaria con 39 casos (19.81\%) o las alteraciones del cordón con 35 casos (15.09\%) (Gráfico 6A). Los otros 160, fueron muertes neonatales precoces (40.82\%) y de estas muertes neonatales, la principal causa fue la asfixia del recién nacido, con 76 casos $(47.60 \%)$; las anomalías congénitas, 35 casos $(21.88 \%)$; y la infección con 32 casos (20\%) (Gráfico 6B).

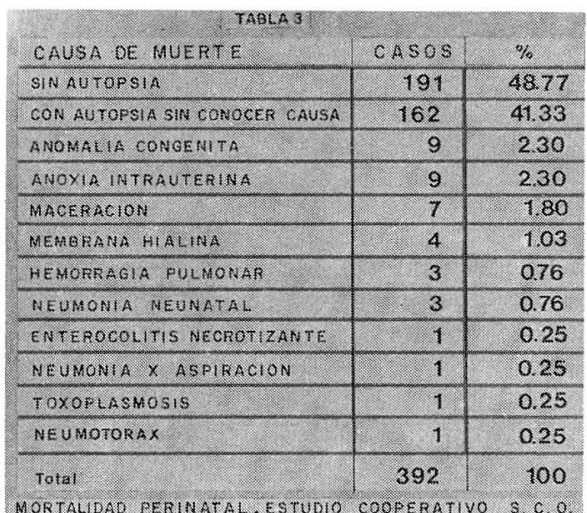

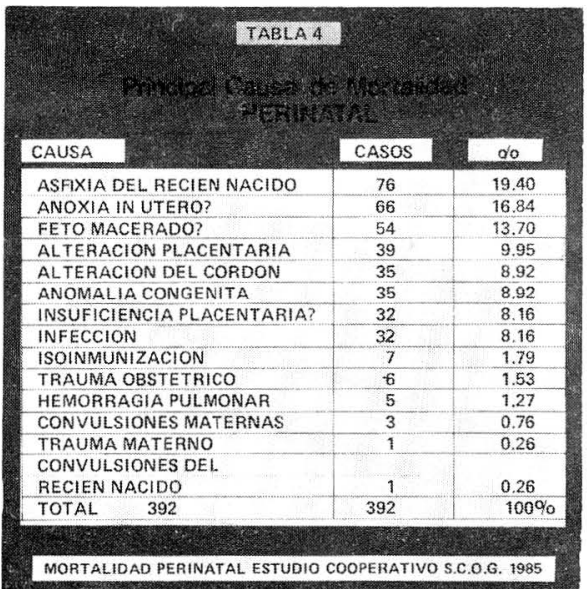



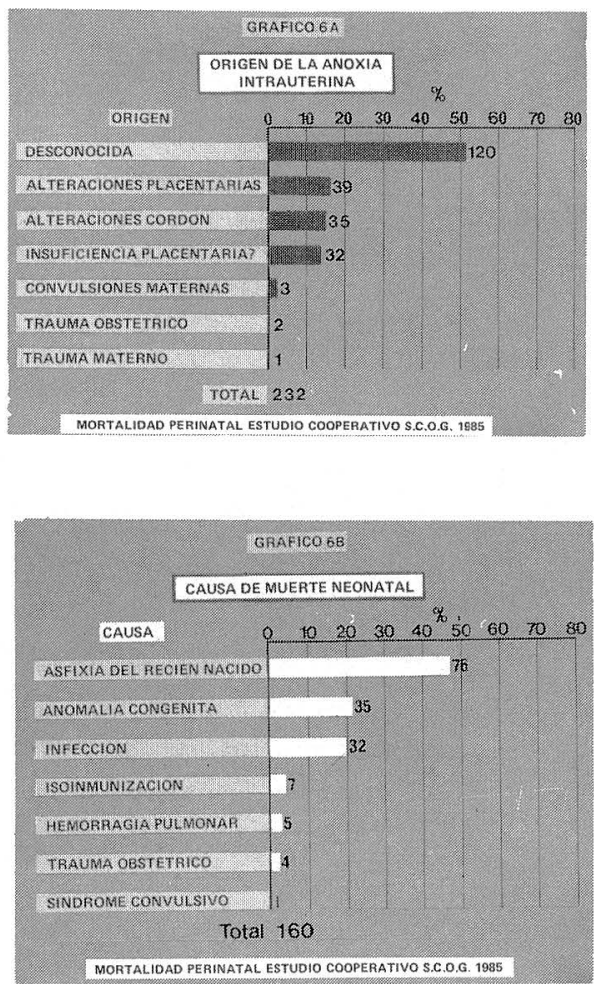

De los 76 casos de asfixia del recién nacido, la gran mayoría, 58 casos, presentaron Síndrome de Dificultad Respiratoria por membrana hialina (76.31 을 (Gráfico 6C).

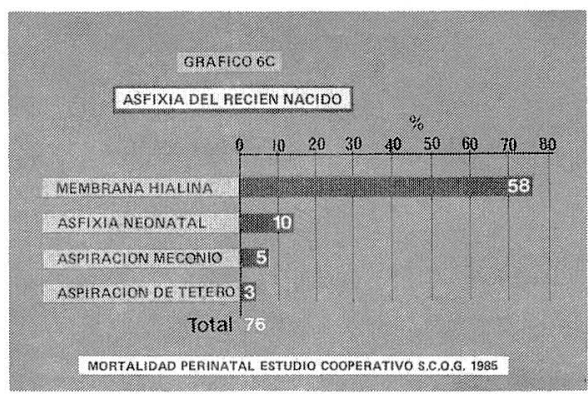

De los 39 casos de origen placentario la patología más frecuente fue el abruptio con 25 casos (64.10\%) (Gráfico 6D).

De los 35 casos debidos a alteración del cordón, la principal causa fue la comprensión, con 18 casos (51.43\%) (Gráfico 6E).

De los 35 casos de anomalías congénitas, las dos principales causas fueron las malformaciones múltiples, con 23 casos $(65.71 \%)$ y la anencefalia con 8 casos (22.85\%) (Gráfico 6F).
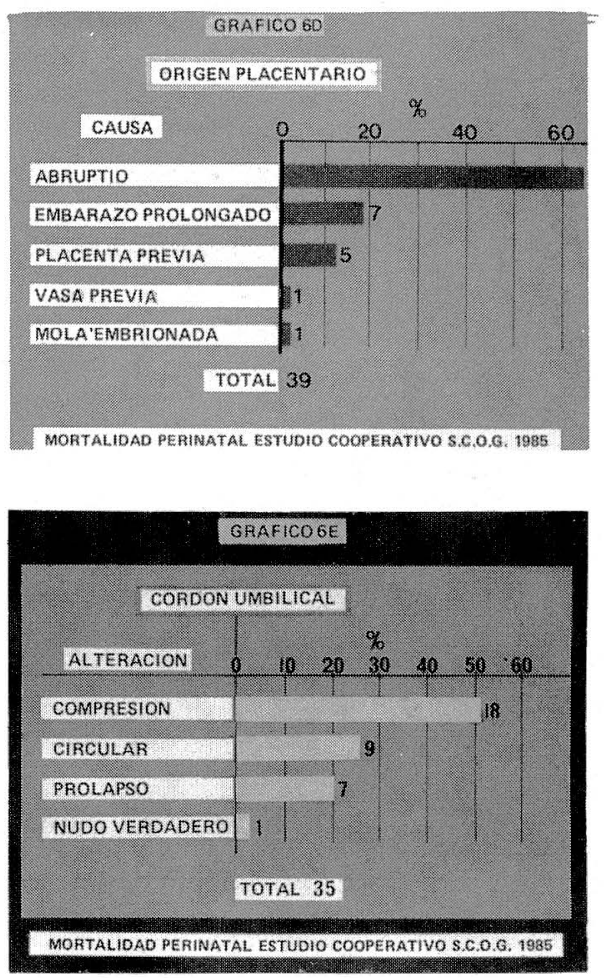

De los 32 casos de infección, las principales causas fueron la sepsis neonatal, con 13 casos (40.63\%), la sepsis in útero con 7 casos (21.87\%), y la sífilis congénita con 7 casos (21.87\%). (Gráfico 6-G). 

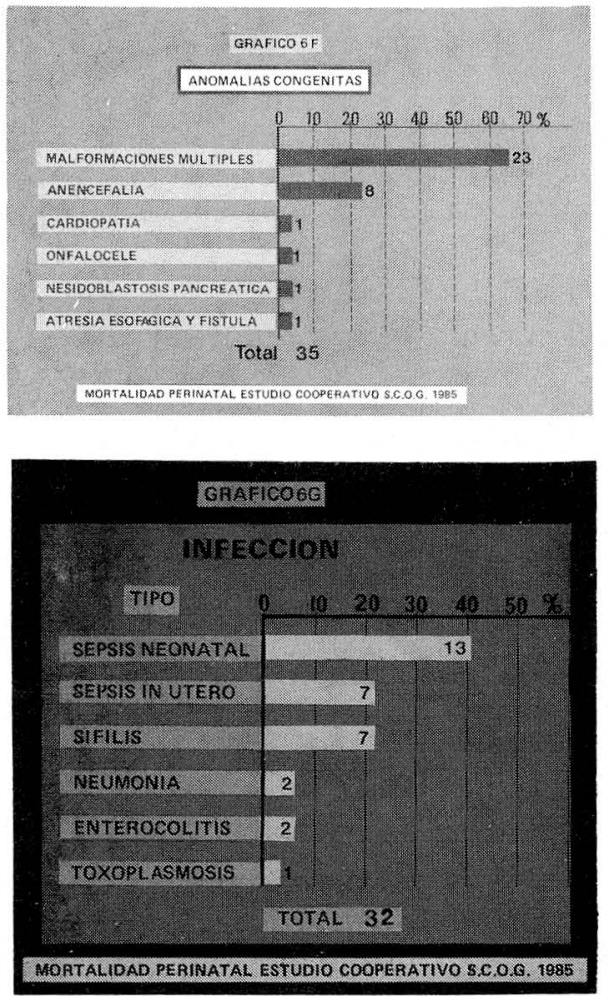

\subsection{Afecciones de la Madre}

No hubo mención de afección materna en 252 casos (64.28\%). De los 180 casos en que se menciona la afección, las más frecuentes fueron: la toxemia, con 74 casos (41.11\%); la afección placentaria con 39 casos (21.67\%); y otras afecciones maternas no relacionadas con el embarazo, con 30 casos. (16.67\%) (Gráfico 7).

\subsection{Momento de la Muerte Perinatal}

La mayoría de los casos se encontraron en el período anteparto, con 175 casos $(44.64 \%)$; y en segundo lugar en el período neonatal, con 160 casos $(40.82 \%)$; la muerte intra-parto sólo aportó 57 casos (14.54\%) (Gráfico 8).

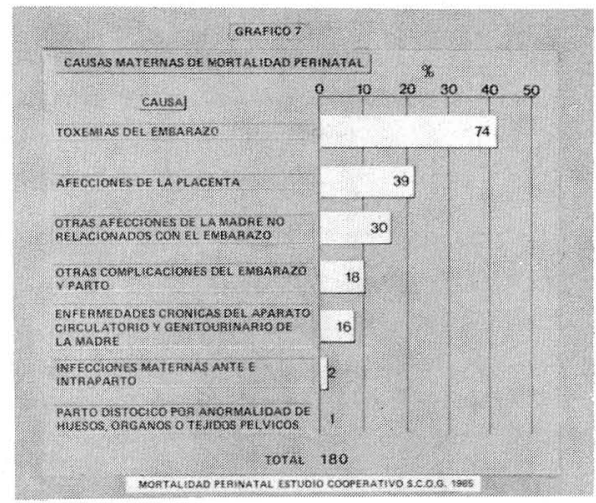

ERAFICO 8

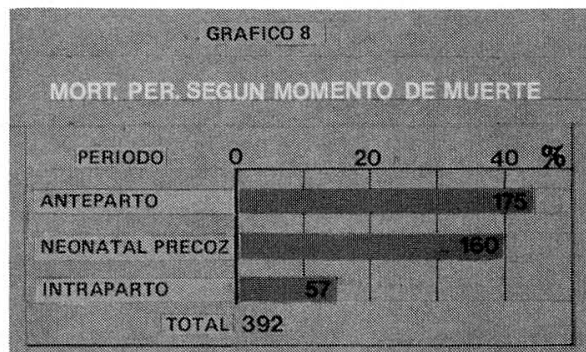

MORTALIDAD PERINATAL. ESTUDIO COOPERATIVO S.C. O.6. 1985

\subsection{Apgar al Minuto}

La mayoría de los casos, 245 (63.64\%), tuvieron Apgar 0 al minuto. Apgar bajo (entre 1 y 6 ), lo tuvieron 94 casos (24.42\%) y Apgar alto (7 y más) lo tuvieron 46 casos (11.94\%). No se clasificó el Apgar al minuto en 7 casos. (Gráfico 9).

\subsection{Apgar a los Cinco Minutos}

La mayoría de los casos, 246 (66.85\%), tuvieron Apgar 0 a los cinco minutos. Apgar bajo (entre 1 y 6 ), lo tuvieron 63 casos (17.12\%); y Apgar alto (7 y más), lo tuvieron 59 casos (16.03\%) (Gráfico 10). No se clasificó el Apgar a los cinco minutos en 24 casos. 

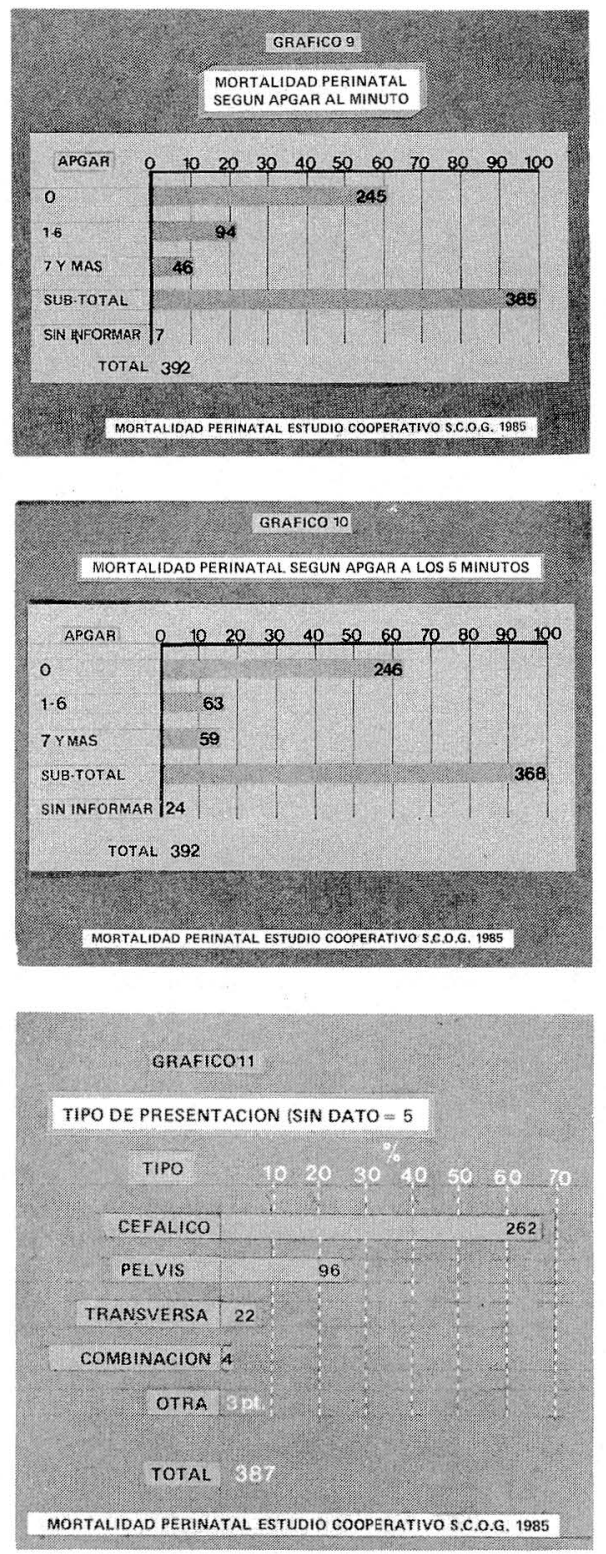

\subsection{Tipo de Presentación}

La mayoría de los casos tenían pre- sentación cefálica, 262 casos (67.70\%); con un gran número de pelvis, 96 casos (24.81\%); y situaciones transversas, 22 casos (5.96\%); no se conoció la presentación en 5 casos (Gráfico 11).

\subsection{Tipo de Parto}

La mayoría fueron espontáneos, 228 casos (58.31 \%); con un gran número de cesárea, 112 casos (28.64\%); y menor número de fórceps o espátulas 37 casos (9.46\%) (Gráfico 12).

\section{GRAFICO 12}

TIPO DE PARTO ( $\sin$ dato 1$)$

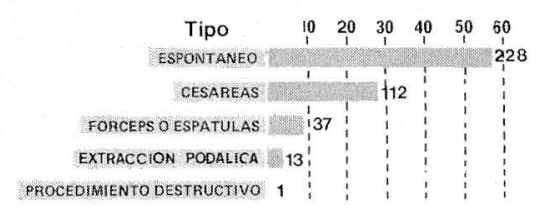

CASOS 39!

MORTALIDAD PERINATAL ESTUDIO COOPERATIVO S.C.O.G. 1985

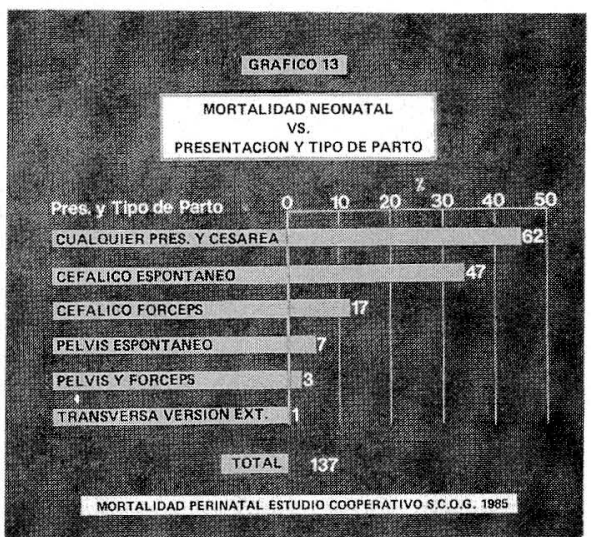

\subsection{Mortalidad Neonatal vs. Presentación y Tipo de Parto}

La mayoría fue cualquier tipo de presentación y cesárea, con 62 casos 
(45.25\%); siguiendo en frecuencia el parto cefálico espontáneo, 47 casos (34.31\%); y el cefálico intervenido con fórceps 17 casos (12.41 \%) (Gráfico 13).

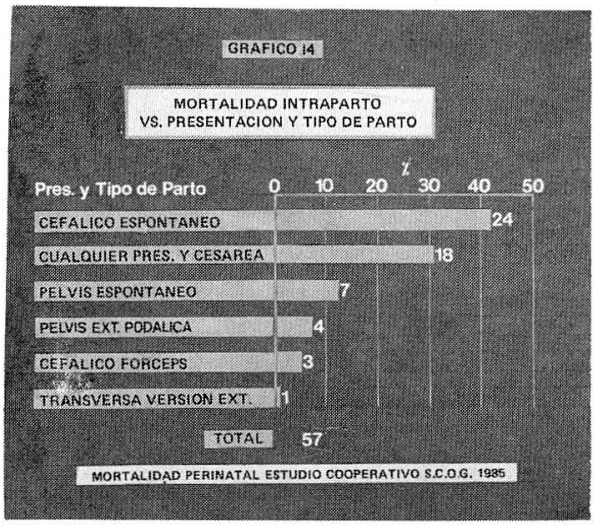

\subsection{Mortalidad Intra-parto Vs.} Presentación y Tipo de Parto

La mayoría fue cefálico espontáneo, con 24 casos (42.11\%); v el segundo lugar cualquier presentación y cesárea, 18 casos (31.58\%); son menos frecuentes el parto de pelvis espontáneo, 7 casos (12.28\%); y el parto de pelvis asistido, 4 casos (7.02\%) (Gráfico 14).

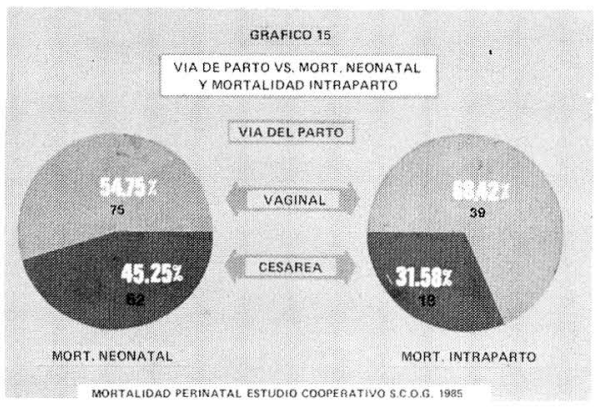

\subsection{Vía del Parto Vs. Mortalidad Neonatal e Intra-parto}

En la mayoría de los casos se encontraron partos por vía vaginal, tanto en la mortalidad neonatal precoz, con 75 casos (54.75\%), como en la mortalidad intra-parto con 39 casos (68.42\%) (Gráfico 15).

\subsection{Duración del trabajo de Parto}

En el mayor número de casos, 138 (36.51 \%), se encontró una duración del trabajo de parto entre 7 y 12 horas. 125 casos (33.07\%), tuvieron un trabajo corto entre 2 y 6 horas, no tuvieron trabajo de parto 44 casos $(11.64 \%)$, trabajo de parto de menos de 2 horas 19 casos (5.03\%) y 52 casos (13.75\%) con un trabajo de parto por encima del promedio, incluso para una primigestante (Tabla 5).

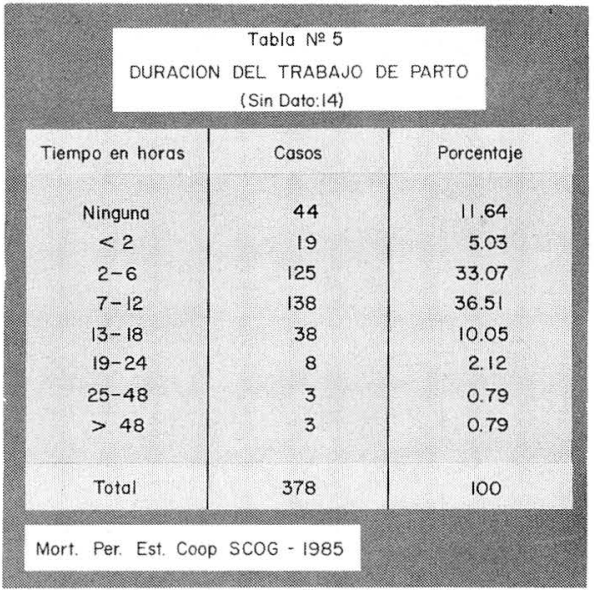

\subsection{Atención del Parto}

La gran mayoría de casos, 279 (71.35\%), fueron atendidos por especialistas en Ginecología y Obstetricia, seguida del estudiante de Medicina que atendió 83 casos (21.23) (Tabla 6).

\subsection{Complicación del Embarazo}

La mayoría de los casos, 156 (39.80\%), no tuvo ninguna complicación durante 
el embarazo. Las complicaciones más frecuentes fueron: los fenómenos hipertensivos del embarazo, con 85 casos $(21.68 \%)$; la ruptura prematura de membranas, con 42 casos (10.71\%); las alteraciones placentarias, 32 casos (8.16\%); las alteraciones del cordón, 18 casos (4.59\%) y los embarazos generales 13 casos (3.32\%) (Tabla 7).
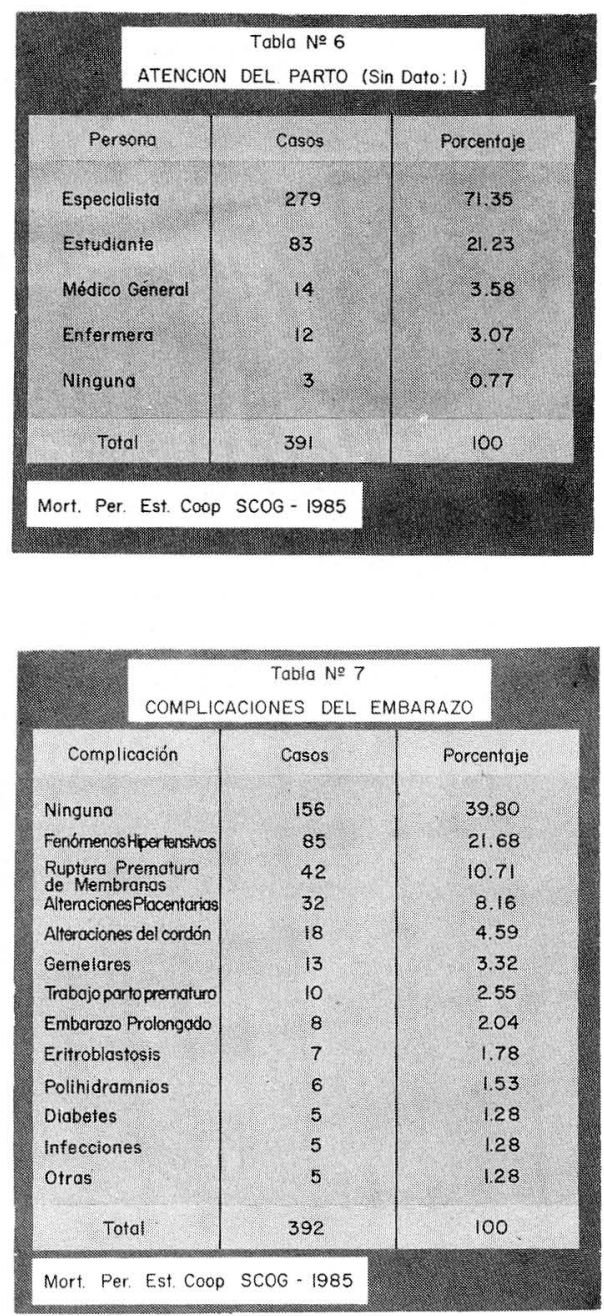

\subsection{Mortalidad Neonatal Precoz Vs. peso y causa principal de Muerte}

Al analizar la mortalidad neonatal precoz, cruzada con el peso del neonato y la causa principal de muerte, encontramos que la membrana hialina es la principal causa, con 63 casos $(60.58 \%)$; siguiendo en frecuencia la anomalía congénita, 13 casos (12.5\%) y la infección, 9 casos (8.65\%).

En el peso adecuado las primeras causas fueron: aspiración de meconio, 4 casos (16\%); la infección, 4 casos (16\%) y el trauma obstétrico, 3 casos (12\%).

Relacionando solamente el peso del neonato, el mayor número de casos lo aportó el bajo peso, 104 (80.62\%), contra el peso adecuado que sólo aporta 25 casos (19.38\%) (Tabla 8).

\section{Tabla $N^{2} 8$}

MORTALIDAD NEONATAL PRECOZ Vs

PESO DEL NEONATO Y CAUSA PRINCIPAL DE MUERTE

\begin{tabular}{l|c|c|c|c|c|c}
\multirow{2}{*}{ CAUSA } & \multicolumn{2}{|c|}{ Bojo Peso } & \multicolumn{2}{c|}{ Peso Adecuodo } & \multicolumn{2}{c}{ Total } \\
\cline { 2 - 7 } & $N$ & $\%$ & $N$ & $\%$ & $N 2$ & $\%$ \\
Membrana Hialina & 63 & 60.58 & 1 & 4.0 & 64 & 49.61 \\
Anomalio Congénito & 13 & 1250 & 3 & 12.0 & 16 & 12.40 \\
Infección & 9 & 8.65 & 4 & 16.0 & 13 & 10.08 \\
Aspiración do Moconio & 3 & 2.89 & 4 & 16.0 & 7 & 5.43 \\
Prematurez & 6 & 5.77 & 0 & - & 6 & 4.65 \\
Trauma Obstétrico & 2 & 1.92 & 3 & 12.0 & 5 & 3.88 \\
Otras & 8 & 7.69 & 10 & 40.0 & 18 & 13.95 \\
\multicolumn{1}{|c|}{ TOTAL } & 104 & 100 & 25 & 100 & 129 & 100 \\
& 104 & 80.62 & 25 & 19.38 & 129 & 100
\end{tabular}

Mort. Per. Est. Coop. SCOG -1985

\section{4 - COMENTARIOS}

A continuación se describen los hechos más importantes, que a juicio de los investigadores ha arrojado este proceso:

4.1. La tasa de mortalidad perinatal es una tasa que refleja en buen grado 
la realidad de la atención perinatal institucional, especialmente la prevista para instituciones de 3er. nivel. Sin embargo, vale la pena resaltar 2 limitantes que son relevantes; la primera el subregistro a que estuvo sometida la prueba en algunos hospitales que por su volumen de atención, no tuvieron la oportunidad de registrar el $100 \%$ de los casos en el período que comprendió el estudio.

La segunda limitante fue la observada en ciertas instituciones que inusitadamente ofrecieron información sensiblemente más baja, sobre todo teniendo en cuenta el grado de riesgo perinatal que manejan, y comparándolas con entidades que atienden igual magnitud, a igual nivel de riesgo.

\subsection{En lo referente a las caracter ísticas} de población materna estudiada se observa lo siguiente:

Edad: Se continúan encontrando proporciones importantes de aquellos hijos de madres que por su edad se consideran de alto riesgo, tales como embarazadas adolescentes, y madres mayores de 35 años.

Paridad: En este aspecto es interesante anotar que la menor proporción de muerte perinatal la está arrojando el grupo de grandes multíparas, quienes a pesar de tener un alto riesgo, por su número cada vez más reducido en la población general, disminuyen el riesgo atribuible a este grupo de presentar el daño.

\subsection{En cuanto al momento de la muerte perinatal, encontramos que la frac- ción de ésta, sucedida en el período intraparto es la de menor proporción, lo cual permita suponer que de alguna ma- nera, la calidad de la atención del parto ha mejorado.}

Ahora bien, dentro de la causalidad específica para el período anteparto, se observa que en la mitad de los casos de muerte intrauterina no se define como causa específica, lo cual comprueba una vez más que este período es de muy difícil clasificación causal, por lo menos con la tecnología disponible que tenemos en nuestro medio; por lo tanto es necesario continuar haciendo énfasis en la vigilancia fetal de pacientes embarazadas que presenten factores con riesgo relativo alto de presentar mortalidad perinatal, con el fin de evitarla o de por lo menos reducirla.

\subsection{En el aspecto de causalidad encon- tramos algunos hechos importantes tales como:}

En general en la mortalidad perinatal, $y$ específicamente en la mortalidad neonatal precoz, la principal causa sigue siendo la dificultad respiratoria del recién nacido, que en el presente estudio fue responsable de la mitad de los casos. Para el efecto es indispensable reforzar el estudio de los factores de riesgo responsables de la iniciación del trabajo de parto pretérmino; así como el análisis cuidadoso de cada caso, en el cual se contemple la posibilidad de interrumpir la gestación antes del término. De igual manera se hace necesario contar con una dotación adecuada en Hospitales del 3er. nivel, desde el punto de vista de atención perinatal, con el fin de tratar de disminuir esta causa de mortalidad.

Dentro de los casos de mortalidad perinatal la presentación de pelvis se encuentra en proporción importante. Si sabemos que este tipo de presentación es más frecuente en los casos con edad gestacional pretermino, que los casos a término, se puede entender este resultado. En nuestro estudio, la población de muertes perinatales en pretérmino fue cercana al $40 \%$.

En la mortalidad neonatal según tipo de parto, observamos que independien- 
te de la presentación, el 45 \% de los neonatos vivos nacieron por cesárea. $\mathrm{Si}$ recordamos que la principal causa de muerte del neonato es el Síndrome de dificultad respiratoria, podemos pensar que la intervención cesárea puede contribuir como causa en esta complicación. A diferencia de la mortalidad neonatal precoz, en la mortalidad intraparto se observa un mayor número de casos con parto vaginal espontáneo, que en cualquier tipo de presentación en la cesárea.

La duración del trabajo de parto por encima del promedio se encuentra un porcentaje apreciable de casos; siendo esta situación un reflejo de calidad en la conducción del trabajo de parto, se plantea la necesidad de recomendar una mejor vigilancia y un adecuado control del mismo.

Observamos en este, como en otros estudios, que la primera causa de complicación materna en el embarazo es la hipertensión inducida por la gestación; causa que ha sido manejada desde el punto de vista materno cada día con mejor tecnología, pero desde el punto de vista peri y neonatal; sigue siendo una causa poco vulnerable, pues a excepción del reposo y las medidas profilácticas una vez instaurado el cuadro, y cuánto más grave sea este, primero el feto $y$ posteriormente el recién nacido, corren un grave riesgo de morbimortalidad.

\subsection{Finalmente, es preciso comentar, que a pesar de que el área de Ana-} tomía Patológica en estos estudios es pilar fundamental, en la presente investigación se constituyó en grave limitante en la búsqueda de causalidad; pues los casos de mortalidad que fueron sometidos a autopsia fueron sólo la mitad y además de las realizadas hay una buena proporción que no aclaró diagnóstico de muerte encontrándose resultados como el de feto macerado, el cual ya se había descrito en los hallazgos clínicos, así como gran número de casos con autop- sia sin conocerse el resultado de la misma.

\section{5 - CONCLUSIONES}

5.1. La tasa de mortalidad perinatal encontrada en el estudio, es superior a la informada por los países industrializados.

\subsection{El Subregistro de este indicador de} salud sigue siendo en nuestro medio importante, constituyéndose en un obstáculo que nos impide conocer cifras reales.

5.3. Las causas más frecuentes de mortalidad perinatal encontradas en nuestro estudio, son causas que pueden reducirse con la aplicación de medidas preventivas y en algunas terapéuticas disponibles en nuestro medio.

\subsection{Sigue siendo una incógnita común} a todas las investigaciones, el establecimiento de causa definida para la mayoría de los casos de mortalidad anteparto.

5.5. La experiencia adquirida en el desarrollo de este estudio, permite concluir que el formulario utilizado en la recolección de la información, puede ser la base, previo ajuste, de un formulario de registro recomendado por FECOLSOG, para ser usado a nivel nacional, con el propósito de mejorar la calidad de la información con respecto a este indicador de salud.

\subsection{La causa principal de muerte neo-} natal precoz en el recién nacido de bajo peso fue la membrana hialina siquiendo en frecuencia la anomalía congénita y la infección. En el recién nacido con peso adecuado las principales causas fueron la aspiración de meconio y la infección siguiendo en frecuencia el trauma obstétrico. 


\section{6 - RECOMENDACIONES}

6.1. Siendo consecuentes con los propósitos que animaron esta investigación, el grupo de trabajo recomienda a las directivas de FECOLSOG, que se pronuncien ante sus sociedades filiales, sobre la necesidad de crear conciencia clara en los integrantes del equipo de salud comprometido en la atención del grupo obstétrico perinatal, del real y verdadero valor, que el indicador "MORTALIDAD PERINATAL" tiene en la evaluación tanto del estado de salud de la población en general, como de la calidad de aten. ción brindada.

6.2. Como estrategia fundamental para cumplir con la anterior recomenda- ción, se propone la conformación de comités operativos institucionales, destinados al estudio de los casos de mortalidad perinatal, integrados por las disciplinas clínicas involucionadas (OBSTE. TRICIA-PEDIATRIA) y por personal de los departamentos de Anatomía Patológica.

6.3. Los resultados obtenidos, deben servir de base en la implementación de programas conjuntos FECOLSOGMINSALUD, enfocados a estandarizar conductas y procedimientos que lleven a disminuir la prevalencia de las causas más frecuentes de mortalidad perinatal. 
Anexo No. 1

SOCIEDAD COLOMBIANA DE OBSTETRICIA Y GINECOLOGIA

ESTUDIO DE MORTALIDAD PERINATAL INSTITUCIONAL EN BOGOTA REGISTRO DE DEFUNCION PERINATAL

Por favor llene las casillas y lineas en blanco

Formulario No.

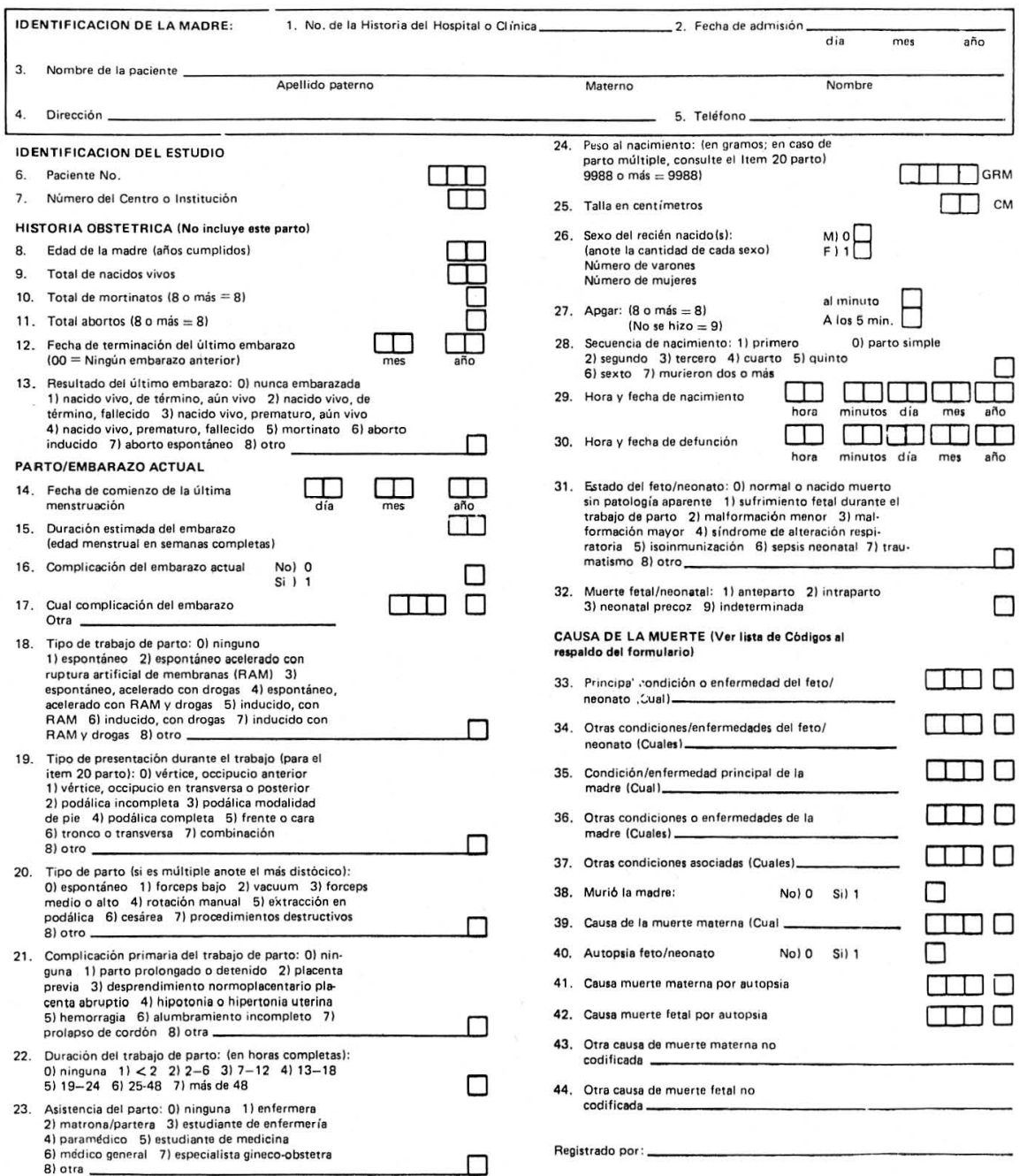

FORMULARIO PRIF MODIFICADO CON LA AUTORIZACION DE LA CCRP DE BOGOTA 


\section{CAUSAS DE MORTALIDAD PERINATALES}

Códigos para Items: 17-33-34-35-36-37-39-41-42

$000.0=$ Ninguna

$$
\text { Grupos de Causas }
$$

Enfermedades crónicas de los aparatos circulatorio y genitourinario de la madre Enfermedad reumática crónica del corazón

Hipertensión crónica

Ofras enfermedades crónicas del aparato circulatorio

Enfermedad crónica del aparato genitourinario

Otras afecciones de la madre no relacionadas con el embarazo

Sifilis

Diabetes mellitus

Rubéola

Traumatismos ocurridos a la madre

Operaciones quirürgicas de la madre

Substancias quimicas transmitidas a través de la placenta

Otras afecciones de la madre

Toxemias del embarazo

Enfermedad renal surgida durante el embarazo

Preeclampsia del embarazo

Eclampsia del embarazo

Toxemia no especificada

Hiperemesis gravidica

Otras toxemias del embarazo

\section{Infecciones maternas ante e intra partum}

Pielitis y pielonefritis del embarazo

Otras infecciones del aparato genitourinario ocurridas durante el embarazo

Otras

Parto distócico por anormalidad de los huesos, organos o tejidos de la pelvis

Con lesión del encéfalo o de la médula espinal, debida al part Con otras lesiones debidas al parto o con las no especificadas Sin mención de lesión debida al parto

Parto distócico por desproporción fetopélvica

Con lesión del encéfalo o de la médula espinal, debida al parto Con otras lesiones debidas al parto o con las no especificadas Con otras lesiones debidas al parto o con
Sin mención de lesión debida al parto

Parto distócico por mala posición del feto

Con lesion del encéfalo o de la médula espinal debida al parto Con otras lesiones debidas al parto o con las no especificadas Sin mención de lesión debida al parto

Parto distócico por anormalidad dinámica del trabajo del parto Con lesión del encéfalo o de la médula espinal, debida al parto Con otras lesiones debidas al parto o con las no especificadas Con otras lesiones debidas al parto a con

Parto distócico por otras complicaciones y por las no especificadas

Con lesión del encéfalo o de la médula espinal, debida al parto Con otras lesiones debidas al parto o con las no especificadas

Sin mención de lesion debida al parto

Otras complicaciones del embarazo y del parto

Incompetencia del cuello uterino

Ruptura prematura de las membranas

Hidramnios

Embarazo ectópico

Embarazo múltiple

Otras complicaciones del embarazo o del parto

Afecciones de la placenta

Placenta previa

Desprendimiento prematuro de la placenta

Infarto de la placenta

Otras afecciones de la placenta

Insuficiencia placentaria no especificada

Afecciones del cordon umbilical

Compresión del cordón umbilical

Prolapso del cordón sin mención de compresión

Otras
Número de la

Lista Detallada

760.2

760.

760.4

761.0
761.1

761.1
761.3

761.5

761.6

761.7

761.2

762.0

762.

762.1

762.2
762.3

762.4

762.5

763.0

763.1

763.1
763.9

764.0

764.2

764.4

765.0

765.2

765.4

766.0

766.2

766.4

767.0

767.0
767.2

767.2
767.4

768.0

768.2

768.4

769.0

769.1

769.2

769.3
769.4

769.5

770.0
770.1

770.1
770.2

770.2
770.8

770.9

771.0

771.1
Grupos de Causas

Número de la

Lista Detallada

Lesión ocurrida durante el embarazo sin mención de causa

Lesión del encéfalo o de la inédula espinal

Otras lesiones debidas al parto y las no especificadas

Interrupción del embarazo

772.0

772.2

773.0

Enfermedad hemolítica del recién nacido

Con incompatibilidad del factor Rh

774.0

Con incompatibilidad de los grupos $A B O$

Con otra incompatibilidad sanguínea o con las no especificadas

Sin mención de causa

774.1

774.2
774.9

Afecciones anóxicas e hipóxicas no clasificadas en otra parte

Enfermedad de membrana hialina y sindrome de dificultad

respiratoria

Anoxia in trauterina

Asfixia del recién nacido, no especificada

Otras afecciones anóxicas e hipóxicas no clasificadas en otra parte

776.1
776.4

776.9

776.0

Otras afecciones del feto y del recién nacido

Inmaturidad no clasificada

Pérdida sangu inea del feto antes del parto

Postmaturidad

Enfermedad hemorrágica del recién nacido

Sindrome causado por el enfriamiento

Otras afecciones

Maceración

Otras muertes fetales de causa desconocida

Anomalías congénitas

Anencefatia

Espina bífida

Otras anomalias congénitas del sistema nervioso y del ojo

Anomalias congénitas del aparato circulatorio

Anomal ias congénitas del aparato respiratorio

Anomalías congénitas del aparato digestivo

Anomalias congénitas del aparato genitourinario

Anomalias congénitas del aparato osteomuscular

Enfermedad de Down

Otras anomalías congénitas que afectan a múltiples aparatos y sistemas

Sistemas
Otras anomalias congénitas y las no especificadas

777.0

778.0

778.1

778.2

778.3

778.9

779.0

740.0

741.0

742.0

743.0

746.0

748.0

749.0

752.0

754.0

759.3

759.0

759.4
745.0

758.0

Infecciones del feto y del recién nacido

Enfermedad diarreica

Listerelosis congénita

Tétan os neonatorum

Septicemia del recién nacido

Enfermedades viricas del RN

Sifilis congénita

Toxoplasmosis congénita

Otras enfermedades infecciosas y parasitarias

009.3

771.2

771.3

771.8

771.1
090.9

771.2

136.9

Otras enfermedades del feto y del recién nacido

Enfermedades de la glándula tiroides congénitas

Fibrosis quística (mucoviscidosis)

Enfermedades de la sangre y de los órganos hematopoyéticos

Neumonia congénita

Muerto in útero por causa desconocida

Sintomas y estados morbosos mal definidos

759.2

277.0

779.6

770.0

779.9

Causas externas de lesiones ocurridas al recién nacido

Calor excesivo

Hambre sed exposición a los elementos yegiguta

900.0

901.0

Aspiración e ingestión de alimentos que provoquen obstrucción sofocación

0 tras causas externas

913.0

994.9 


\section{ANEXO 2}

\section{INSTRUCTIVO DEL REGISTRO DE DEFUNCION PERINATAL NORMAS GENERALES}

\section{Caso}

1.1. Defunción perinatal es toda muerte perinatal ocurrida desde la semana 28 de gestación y/o 1.000 gramos o más de peso (Mortalidad Fetal Tardía) y las muertes Neonatales ocurridas hasta el 7o. día de nacido (Neonatal Precoz).

1.2. Las semanas de gestación: se contarán a partir del 1er. día de la última regla.

1.3. En caso de no conocer la fecha de la última regla se registrarán los fetos mortinatos de 1.000 gramos o más.

1.4. Todo caso ocurrido que no se encuentre dentro de los parámetros

1.1 a 1.3 no debe incluirse en el estudio.

\section{Normas para el Registro de la Información}

2.1. Las casillas deben ser llenadas con números arábigos en todos los casos y nunca deben ser ocupadas por la letra $X$.

2.2. No deben dejarse casillas en blanco.

2.3. Cuando en la variable exista más de una casilla por ejemplo el ITEM No. $6 \square \square \square$ y la información consta de un solo dígito (caso No. 8), esta cifra debe colocarse en la casilla de la derecha y las restantes casillas deben llenarse con ceros así:

2.4. Anotar siempre el código correspondiente, que se encuentra antes del paréntesis y no los números que contiene la variable; ejemplo: Duración del trabajo de parto (en horas completas).

0) Ninguna
1) $<2$
2) $2-6$
3) $7-12$
4) $13-18$
5) $19-24$
6) $25-48$
7) más de 48

Si la paciente presentó su trabajo de parto de 8 horas, se registrará así: 3 y no $7-12$

\section{Cuándo se debe hacer el registro de la información}

El registro de la información en el formulario, debe hacerse, cuando se conozcan todos los datos que solicita el mismo; de esta manera el caso estará completo.

Para casos especiales cuando falte información de alguna variable, deberá consultarse con el médico responsable de la investigación en la institución. (coordinador).

\section{Quién debe investigar la información}

Deberá ser hecho, el registro por la o las personas encargadas de la investigación en cada institución. (coordinador o por quien él designe).

\section{De la identificación}

a. Del formulario: cada formulario por utilizar estará previamente numerado.

b. De la Madre:

\section{Número de la Historia del Hospital} o Clínica: anotar el número corres- 
pondiente a la historia clínica de la Madre en la institución.

2. Fecha de admisión: Anotar el día, mes $y$ año de ingreso de la paciente a la institución. Utilizar para el registro números arábigos.

3. Nombre de la paciente: Anotar en su orden, apellido paterno, apellido materno, o de casada, y nombre o nombres completos.

4. Dirección: Anotar, la dirección, localidad (barrio, vereda), municipio y teléfono donde reside la paciente, la mayor parte del tiempo. La dirección, para este efecto, es sinónimo de residencia habitual.

c. Del estudio:

5. Paciente No.: Anotar el número de paciente que corresponde, según numeración consecutiva llevada en libro de control en cada institución.

6. Número de Centro o Institución: Anotar el código correspondiente a la institución, previamente asignado por el estudio en la siguiente forma:

Materno Infantil 01

Hospital Militar Central 02

\section{7 - BIBLIOGRAFIA}

1. ABUDU O. AKINKUBE A. "Clinical causes and classification of perinatal mor. tality in lagos". Int. J. Gynaecol Obstet. 1982. Dec; 20 (6): $443-7$.

2. BALLAS S. HORNSTEIN E: JAFFA AJ. TOAFF R. "Selective versus Routine in trapartum monitoring: Comparison of effects on perinatal outcome". Acta Obstet Gynecol scand 1980; 59 (4): 301-4.

$\begin{array}{ll}\text { Hospital Simón Bolívar } & 03 \\ \text { Hospital San Ignacio } & 04 \\ \text { Hospital San Blas } & 05 \\ \text { Clínica Palermo } & 06 \\ \text { Hospital San José } & 07 \\ \text { Hospital Infantil } & 08 \\ \text { Centro Médico de los Andes } & 09 \\ \text { Clínica David Restrepo } & 10 \\ \text { Hospital de la Samaritana } & 11\end{array}$

\section{Causa de la Muerte:}

En este ITEM, las casillas de la derecha están destinadas para anotar el número correspondiente a la causa principal y otros, de muerte perinatal o materna; este número se encuentra al respaldo del formulario según causa, ejemplo:

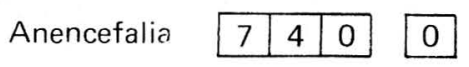

7. En el ITEM, 17 "Cuál complicación del embarazo" utilice también los códigos al respaldo del formulario.

Ejemplo:

Embarazo Múltiple \begin{tabular}{|l|l|l|}
\hline 7 & 6 & 9 \\
\hline
\end{tabular}

3. BARNS T. "Obstetrics in the third world with particular reference to field. research into delivery of maternal care to the community". En: Bourne $\mathrm{C}$, en: Recent advances in Obstetrics and Gynecology. Edimburgh: London, 1979; Churchill. Livingstone.

4. BOWES WA Jr. "A review of perinatal. mortality in Colorado, 1971 to 1978, and. its relationship to the regionalization of 
perinatal services". Am. J. Obstet Gynecol 1981 Dec. 15; 141 (8): 1045-52.

5. BOYLAN P.O. DRISCOLL K. "Improvement in perinatal mortality rate attribu. ted to spontaneous preterm labor without use tocolytic Agents". Am. J. Obstet Gynecol 1983 Apr 1; 145 (7): 781-3.

6. CHRISTENSEN K.K. "Infection as a predominant cause of perinatal mortality". Obstet Gynecol 1982 Apr; 59 (4): 499-508.

7. “Compendio de estadísticas vitales en México: México: Secretaría de Salubridad y Asistencia, 1976".

8. DAIKOKU NH; KALTREIDER DF; JOHNSON TR Jr; JOHNSON JW; SIMNONS MA. "Premature rupture of membranes and preterm labor: Infection and perinatal mortality risks". Am. J. Public Health 1981 JAN; 71 (1): 38-46.

9. DIAZ R. "Mortalidad Perinatal en Bogotá, 1983 - 1984 Secretaría de Salud del Distrito Especial de Bogotá, Colombia, Cifras oficiales de certificados de defunción y de Natalidad. En proceso de Publicación Rev. Col. Obst. y Gin.

10. EFFER SB; SAIGAL S; RAND C; HUNTER DJ; STOSKOPF B; HARPER AC; NIMROD C, MILNER R. "Effect of Delivery method on outcomes in the very lowbirth weight breech infant: is the improved survival related to cesarea section or other perinatal care maneuvers". Am J. Obstet Gynecol 1983 JAN 15; 145 (2): 123-8.

11. ERKOLA R; KERO P; SEPP "AL" $A$; GR'ONROOS M; RAURAMO L. "Monitoring perinatal Mortality by birth weight specific Mortality rates". Int J. Gynaecol Obstet 1982 Jun; 20 (3); 231.5

12. FABIA J; DROLETTE M. "Twin Pairs, smoking in pregnancy and perinatal mor- tality". Am J. Epidemiol 1980 Sept; 112 (3): 404-8.

13. FAUNDES A; HARDY E; DIAZ J; PINOTTI J. "Association of marital status and years of schooling with perinatal outcome; the influence of prenatal care as an intermediate variable". J. Perinat Med. 1982; $10(2)$ : 105-13.

14. FORBES JF; BODDY FA; PICKERING R; WYLLIE MM. "Perinatal mortality in Scotland: 1970-9". J. Epidemiol Community Health 1982 Dec; 36 (4): 282-8.

15. FOSTER FH. "Changes in social and biological factors in New Zeland perinatal mortality". N Z Med J. 1982 Sept. 22; 95 (716): 657-9.

16. HOSKINS EM ELIIOT E; SHENNAN AT; SKINDMORE MB; KEITH E. "Outcome of very low-birth weight infants born at a Perinatal Center". Am J. Obstet Gynecol 1983 JAN 15; 145 (2): 135-40.

17. INSULL B J; MAC LEAN NE; BLANCHETTE G. "Perinatal Mortality in Southland". N Z med J. 1980 JAN 23; 91 (652): 40-2.

18. ITSKOVITZ J; ABRANOVICI H; BRANDES JM. "Oligohydramnion. Meconium and perinatal death concurrent with indomethacin treatment in human pregnancy". J. Reprod Med 1980; 24 (3): 137-40.

19. KAFATOS AG; PANTELAKIS SN. "Factors related to perinatal morbidity and mortality Risk factors in pregnancy influencing perinatal morbidity and mortality". Pediatrician 1982; 11 (2): 27-44.

20. KASS E.H. "Infectious disease and perinatal morbidity". Yale J. Biol med 1982. May-Aug; 55 (3-4): 231-7.

21. KNUTZEN VK; SHER G. "The major determinants of perinatal Mortality in a 
Large Metropolitan Hospital. Fiesults of a retrospective study". J Reprod med. 1982 Jul; 27 (7); 395-400.

22. KUNDSIN RB; DRISCOLL SG; PELLE. TIER PA. "Ureaplasma urealyticum in. criminated in perinatal Morbidity and mortality". Science 1981 Jul 24; 213 (4506): $474-5$.

23. LANGER A; ARROYO P. "La mortalidad perinatal en el Instituto Nacional de Perinatología, México". Bol Med Hosp., Infant Mex. Vol 40 número 11 Nov. 1983.

24. LAW SW 2d; GIBBONS W; POINDEXTER AN. "Patient coperation: A Determinant of perinatal outcome in the prehant diabetic. J. reprod. Med. 1980 May; 24 (5): 197-201.

25. LEE K; TSENG; P; EIDELMAN A; KAN DALL S; GARTNER L. "Determinants of the neonatal mortality". Am. J. Dis Child 1976; 130: 842.

26. LEIV S; BAKKETEIG; HOFFMAN HJ. "Tendencia a repetir edad Gestacional y peso en partos sucesivos, relacionado con sobrevida perinatal". Acta Obstet Gynecol Scand 1983; 62: 385-392.

27. LINDGREN L. "Perinatal mortality and different socioeconomic status" Acta Obstet Gynecol Scand 1981; 60 (1): 21-6.

28. L' OFGREN O; POLBERGER S. "Perinatal mortality: Changes in the diagnostic panorama 1974-1980". Acta Pediatr Scand 1983 May: 72 (3): 327-32

29. LOPEZ G'; RIAÑO G. "Atención del parto hospitalario en Colombia primeros hallazgos". Monografías de la CCRP: Vol. 11, junio 1979.

30. MARTIN DH; KOUTSKY L; ESCHENBACH DA; DALING JR; ALEXANDER
ER; BENEDETTI; JK; HOLMES KK. "Prematurity and perinatal mortality in pregnancies complicated by Maternal Chlamydia trachomatis Infections". JAMA 1982 Marz. 19, 247 (11): 1585-8.

31. NAEYE RL. "Abruptio placentae and placenta previa: Frecuency, perinatal mortality, and cigarette smoking". Obstet Gynecol 1980 Jun; 55 (6): 701-4.

32. OMS. "Clasificación Internacional de Enfermedades".

33. O' DRISCOLL K; FOLEY M. “Correlation of decrease in perinatal mortality and increas in cesarean section rates". Obstet Gynecol 1983 JAN; 6 (1): 1-5.

34. OUNSTED M; MOAR V; SCOTT WA. "Perinatal Morbidity and mortality in small for dates babies the relative importance of some maternal factores". Early hum deu 1981 Sep: 5 (4): 367-75.

35. PAUL RH; G' AUTHIER RJ; QUILLI. GAN EJ. "Clinical fetal monitoring. The usage and relationship to trends in cesa. rean delivery and perinatal mortality". Acta Obstet Gynecol Scand 1980; 59 (4) : 289-95.

36. PEARSON JW. "Cesarean section and perinatal mortality $A$ nine year experience in a city county Hospital" Am J. Obstet Gynecol 1984 JAN 15; 148 (2), 155-9.

37. PERKINS RP. "The neonatal significance of selected perinatal events. Among infants of low birth weight II. The influence of Ruptured membranes". Am. J. Obstet Gynecol 1982 JAN 1; 142 (1): 7-16.

38. PUISSANT F; LEORY F. "A reappraisal of perinatal mortality factores in Twins" Acta Genet med Genellol (Roma) 1982; $31(3-4): 213-9$ 
39. Salud Perinatal: Boletín del Centro Latino Americano de Perinatología y Desarrollo Humano Vol. 2 No. 41985.

40. SEHGAL NN. "Perinatal mortality in twin pregnancies implications for Clinical Management". Fostgrad med 1980. Nov; 68 (5): 231, 233, 236.

41. STANLEY FJ; HOBBS MS. "Perinatal outcome in western Australia 1968 to 1975; Social and biological determinants. Med. J. aust 1981.

42. STERN L. "The value of effectiveness of neonatal intensive care; Its relationship to perinatal mortality and morbidity". Pediatr Padol.1982; 17 (2); 245-57.

43. STUBLEFIELD PG; BEREK JS. "Perinatal mortality in term and post-term births". Obstet Gynecol 1980 Dec; 56 (6); 676-82.
44. TERRIN M; MEYER MB. "Birth weight sepcific rates as a bias in the effects of Smoking and other perinatal hazards". Obstet Gynecol 1981 Nov; 58 (5): 636-8.

45. WILLIAMS RL; CHEN PM. "Identifyng the sources of the recent decline in perinatal mortality rates in CALIFORNIA". N Engl J. med 1982 JAN 28, 306, (4);

46. WILLIAMS RL; CREASY RK; CUNNINGHAN GC; HAWES WE; NORRIS FD; TASHIRO M. Fetal Growth and perinatal viability in California" Obstet. Gynecol 1982 May; 59 (5): 62432 :

47. YLINEN K; RAIVIO K; TERAMO K. Haemoglogin Alc predicts the perinatal outcome in insulin Dependent diabetic pregnancies". 